\title{
ChainCQG: Flow-Aware Conversational Question Generation
}

\author{
Jing Gu ${ }^{1,3}$ Mostafa Mirshekari $^{1}$ Zhou Yu $^{1,2}$ Aaron Sisto $^{1}$ \\ ${ }^{1}$ Searchable.ai \\ ${ }^{2}$ Columbia University \\ ${ }^{3}$ University of California, Davis \\ \{jing,mostafa,aaron\}@searchable.ai \\ zy2461@columbia.edu
}

\begin{abstract}
Conversational systems enable numerous valuable applications, and question-answering is an important component underlying many of these. However, conversational questionanswering remains challenging due to the lack of realistic, domain-specific training data. Inspired by this bottleneck, we focus on conversational question generation as a means to generate synthetic conversations for training and evaluation purposes. We present a number of novel strategies to improve conversational flow and accommodate varying question types and overall fluidity. Specifically, we design ChainCQG as a two-stage architecture that learns question-answer representations across multiple dialogue turns using a flow propagation training strategy. Chain $C Q G$ significantly outperforms both answer-aware and answer-unaware SOTA baselines (e.g., up to $48 \%$ BLEU-1 improvement). Additionally, our model is able to generate different types of questions, with improved fluidity and coreference alignment.
\end{abstract}

\section{Introduction}

Conversational systems are important in many realworld applications, including personal assistants, educational tutors (Winkler et al., 2020), customer service (El Asri et al., 2017; Budzianowski et al., 2018), and increasingly, entertainment. A key component of these systems is the ability to interpret a search query and retrieve information from different sources as naturally and efficiently as possible. In analogous human interactions, such a search generally occurs through conversation. In this context, a conversation consists of a sequence of dialogue turns during which the search objective becomes clearer over time. The applications mentioned above could benefit greatly from this type of multi-turn interaction, enabling conversational agents to accurately predict intent, request additional information, and better understand ambiguous followup questions and comments. In an applied setting, meaningful and natural conversations are important features of virtual entities as a means to establish trust and improve usability.

Here, we are motivated by the challenging task of conversational question answering (CQA). Current open-source datasets such as CoQA (Reddy et al., 2019) and QuAC (Choi et al., 2018) provide strong baselines for this task. However, these datasets have limited applicability in practical settings, because 1) they are created from domainagnostic source material, and 2) they do not necessarily consider the full diversity of question types and vernacular that may be encountered in natural dialogue. Creating realistic, domain-specific datasets to train CQA models is notoriously costly and time-consuming. As such, we focus on the related task of question generation as a means to generate synthetic conversational questions and subsequently, create new datasets or augment existing ones. This will ultimately enable training CQA models in closed-loop, simulation environments, as well as allow machines to initiate dialogue and engage in information-seeking behavior.

While QA models have been studied previously (Zhu et al., 2018; Huang et al., 2019; Yeh and Chen, 2019; Chen et al., 2020; Ju et al., 2019; Ohsugi et al., 2019)), the QG task, which is the focus of this paper, has received less attention. QG models in the answer-unaware setting aim to predict a question given the source passage, while in the answer-aware setting, the target answer and rationale are included as inputs as well. Most QG-related literature has focused on single-turn question generation using question-answer datasets such as SQuAD (Rajpurkar et al., 2016), and other textual sources like Wikipedia articles (Du and Cardie, 2018).

Conversational Question Generation (CQG) 
proves more challenging than single-turn QG as the questions are often highly ambiguous on their own, forcing the model to learn a deeper understanding of the context surrounding the passage text and dialogue history (Pan et al., 2019). Most CQG studies have generated questions using only the passage and dialogue history as inputs (i.e., answerunaware) (Pan et al., 2019; Qi et al., 2020; Nakanishi et al., 2019; Wang et al., 2018). Answer-aware CQG models, on the other hand, generate questions based on the target answer, as well as dialogue history and passage. Although answer-aware CQG models seek to improve the generated conversation flow, current answer-aware QG models suffer from issues including inaccurate coreference alignment, dialogue inconsistencies, incorrect grammar and the inability to generate many different types of questions (e.g. yes/no, factoid, explanation).

In this paper, we introduce ChainCQG, a Conversational QG model that achieves improved performance by jointly learning the representations of questions and answers sequentially, across multiple dialogue turns. To this end, we outline a two-stage architecture, inspired by the approach discussed in $\mathrm{Wu}$ et al. (2019) and Gu et al. (2020), where two language models are used to simulate user and system in a response generation task. Our ChainCQG model is trained end-to-end, resulting in high-quality questions, while reducing computational cost by using shared parameters across both models. Using an answer-aware strategy grounds each turn of QG by jointly encoding the passage with the target answer rationale, increasing accuracy of the generated question types and further aligning coreferences between dialogue turns. We evaluate our approach using the inverted CoQA dataset (Reddy et al., 2019), which is a large-scale CQA dataset that we re-purposed for question generation. Our model outperforms existing SOTA CFNet (Gao et al., 2019) and ReDR (Pan et al., 2019) by a large margin on automatic evaluation metrics, and shows improved results on human evaluation metrics as well. More information about the baselines will be discussed in Section 2 .

In summary, the main contributions of this paper are threefold ${ }^{1}$ :

- The Chain $C Q G$ two-stage architecture is introduced with answer-aware input encoding, and it is an end-to-end model which is able to

\footnotetext{
${ }^{1}$ Code available at https://github.com/ searchableai/ChainCQG.
}

fluently generate different types of questions and achieve high consistency with the target answers.

- We demonstrate a flow propagation-based training method to learn question-answer representations across multiple dialogue spans.

- ChainCQG sets the new SOTA results on the answer-aware CQG task with robust human evaluation results.

The remainder of the paper is structured as follows: First, we discuss related work and how our approach is distinguished from previous methods (in Section 2). Then, we discuss the ChainCQG framework and preprocessing steps (in Section 3). Next, we describe our experiments, datasets and metrics, and evaluation results (in Section 4). Finally, we discuss conclusions, future work and the ethical issues (in Sections 5 and 6).

\section{Related Work}

In this section, we first explore previous approaches to question generation and then discuss outstanding research challenges in conversational question generation.

\subsection{Single-turn Question Generation}

Single-turn question generation has been the focus of extensive research. Two of the main categories in QG are answer-unaware and answeraware. The former category generates the question without knowledge of the answer and solely based on the passage; whereas, the latter takes both passage and answer as inputs. Traditional approaches for answer-unaware QG include two main steps: content selection and question generation (Du and Cardie, 2017; Subramanian et al., 2018). Some of the more recent approaches utilize sequence-to-sequence (seq2seq) models for end-toend question generation using Transformer-based architectures (Scialom et al., 2019). Various techniques have been used for improving the generated questions, including contextualized word embeddings (Scialom et al., 2019), question type usage and copying mechanism (Wu et al., 2020), and typed decoders (Wang et al., 2018).

To enable answer-aware question generation, the input passage is augmented with information describing the answer. For example, the passage can be concatenated with the answer positions and 
lexical features (e.g., part-of-speech (POS) and named entity (NER)) to form the encoder input of a seq2seq model (Zhou et al., 2017). Jointly modelling the unstructured passage and the structured answer-relevant relation has been suggested for improving question generation as well (Li et al., 2019). Additional techniques have been proposed to solve various answer-aware QG challenges, including poor performance on long passages (Zhao et al., 2018) and the bias of repeating the terms in the target answer within the generated question (Kim et al., 2019).

\subsection{Conversational Question Generation}

Compared to single-turn QG, conversational (i.e., multi-turn) QG is less frequently explored in the literature. Further, it is more difficult as it requires a deeper understanding of the context and the dialogue history. Previous work mostly focused on answer-unaware CQG (Pan et al., 2019; Qi et al., 2020; Nakanishi et al., 2019; Wang et al., 2018). Specifically, Pan et al. (2019) proposed an encoderdecoder framework, ReDR, for answer-agnostic $\mathrm{CQG}$, which is fine-tuned using feedback from an independent question-answer model. However, in this setting, maintaining conversational flow and consistency between dialogue turns is a primary challenge.

In this paper, we focus on answer-aware question generation. By grounding the generated question with the target answer rationale in each turn, this approach seeks to improve conversational flow and question-answer consistency. Within answeraware CQG, (Gao et al., 2019) introduced the current SOTA, CFNet, which combined an auxiliary coreference alignment module with a copy mechanism and dialogue flow embedding. As will be described (in Section 4), we compare our model to answer-aware, CFNet (Gao et al., 2019), and answer-unaware, ReDR (Pan et al., 2019), SOTA baselines. As a practical note, in real-world applications, answer-aware QG systems may be augmented with an Answer Generation (AG) model to form an answer-unaware model that predicts the next conversational question-answer pair jointly. Discussing this AG model is out of the scope of this paper and will be the focus of future work.

\section{The ChainCQG Framework}

Chain $C Q G$ learns the question-answer representations jointly using two modules: Answer Encod- ing (AE) and Question Generation (QG). Encoding the answer based on the passage and dialogue history improves the answer understanding within the QG module, which in turn improves the generated questions. In the rest of this section, we provide a description of the input pre-processing steps, the general CQG problem formulation, and the $\mathrm{AE}$ and QG modules.

\subsection{Task Definition}

The conversational QG task in this paper aims to predict the next question given the passage $(\mathrm{P})$, target answer $\left(A_{n}\right)$ and history of the dialogue preceding the $\mathrm{n}^{\text {th }}$ turn, $\left(H_{n}\right)$. We also consider the answer rationale in each turn, and annotate the passage with the target answer rationale span, which we denote as $P_{H L_{n}}$. Mathematically, given the annotated passage $\left(P_{H L_{n}}\right)$, target answer $\left(A_{n}\right)$, and dialogue history $\left(H_{n}=\right.$ $\left.\left(\left(Q_{1}, A_{1}\right),\left(Q_{2}, A_{2}\right), \ldots,\left(Q_{n-1}, A_{n-1}\right)\right)\right)$, the $\mathrm{QG}$ task predicts the next question $Q_{n}$. This task can be defined explicitly as generating a question, $\hat{Q}$, where:

$$
\hat{Q}=\underset{Q_{n}}{\operatorname{argmax}} \operatorname{Prob}\left(Q_{n} \mid P_{H L_{n}}, A_{n}, H_{n}\right) .
$$

\subsection{Input Preprocessing}

In this Section, we briefly describe the processing steps necessary to prepare the input data. Specifically, we take the following approach:

1. We first create $n$ sub-dialogues based on the full dialogue, with the $i$-th sub-dialogue starting from the first turn and finishing with the $i$ th turn, i.e., $S D_{1}=\left\{\left\{Q_{1}, A_{1}\right\}\right\}, S D_{2}=\left\{\left\{Q_{1}\right.\right.$, $\left.\left.A_{1}\right\},\left\{Q_{2}, A_{2}\right\}\right\}, \ldots, S D_{n}=\left\{\left\{Q_{1}, A_{1}\right\},\left\{Q_{2}\right.\right.$, $\left.\left.A_{2}\right\}, \ldots,\left\{Q_{n}, A_{n}\right\}\right\}$.

2. For the $i$-th sub-dialogue, we use a highlight token, [HL], to denote the answer rationale in the passage corresponding to the answer in the $i$-th turn, which serves as additional context for the target answer.

3. For each sub-dialogue, $i$, the passage (with the highlighted token corresponding to the $i$ th turn) is concatenated with the first answer (i.e., $A_{1}$ ) by a SEP token. We denote the concatenation as $A^{*}$.

4. We then reverse the order of the answers and questions in the sub-dialogues (e.g., $\{\mathrm{A} 1, \mathrm{Q} 1\}$ instead of $\{\mathrm{Q} 1, \mathrm{~A} 1\})$. The reason behind this 


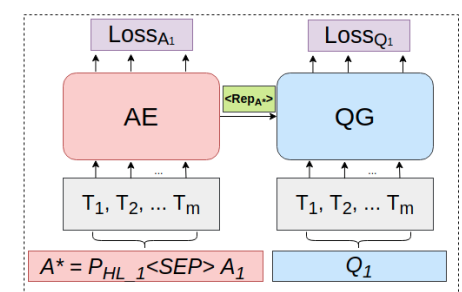

a) First Turn
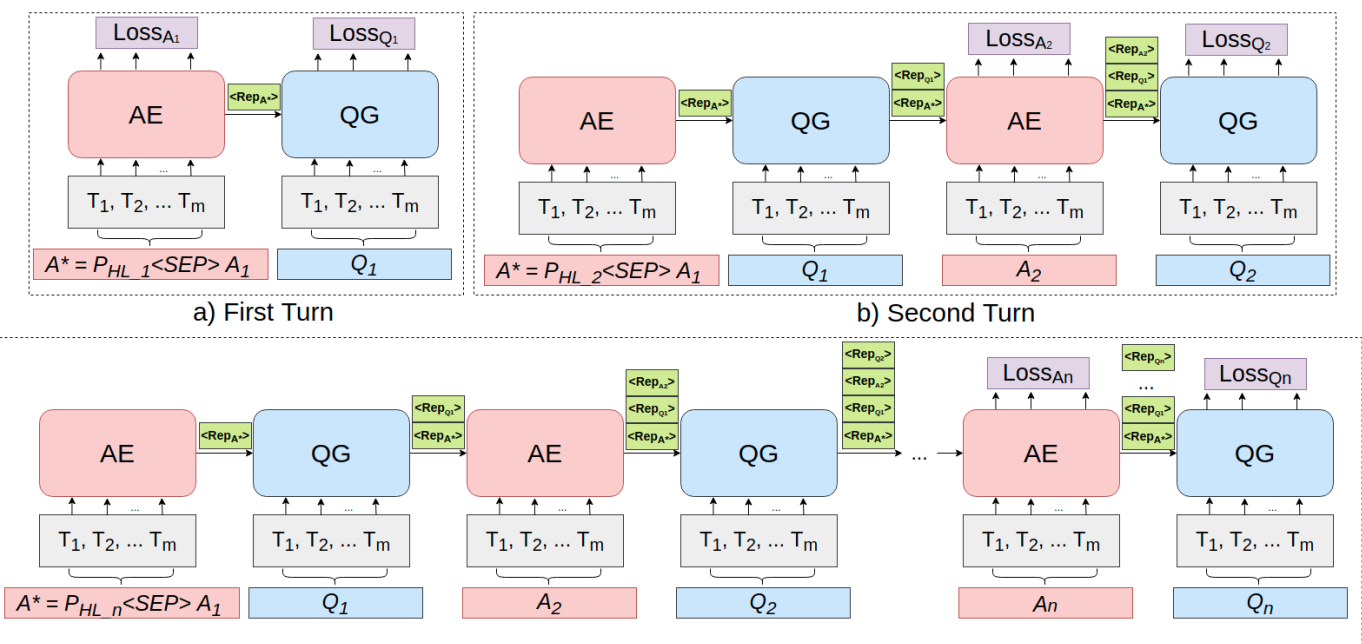

c) N-th Turn

Figure 1: Main structure of ChainCQG. Each QA turn in the dialogue span is trained with a separate conversational flow that contains all previous dialogue turns. Answer Encoder and Question Generator modules iteratively generate and share answer and question representations across multiple dialogue turns.

step is to align the input sequence with the natural order of the QG task, where the questions come after the answers. We examine the effects of this ordering scheme in later ablation studies.

\subsection{Answer Encoding and Question Generation Modules}

In this paper, we use GPT-2 (Radford et al., 2019) to represent both the $\mathrm{AE}$ and $\mathrm{QG}$ modules described above. Specifically, the AE model is used to learn the representation of the passage and answer in each turn, and the QG model is used to learn the representation of the dialogue history and generate the next question in the conversation. The AE and QG models communicate via the models' hidden states, which are the $\mathrm{K}$ and $\mathrm{V}$ values when using GPT. $\mathrm{K}$ and V values together form a contexual representation for the entire conversation history.

\subsection{Flow Propagation-Based Training}

To improve the conversational flow of the CQG, we introduce a sequential training process. Figure 1 shows the main structure. To make it more concrete, let us consider a dialogue of $n$ turns ( $\left\{\left\{Q_{1}\right.\right.$, $\left.\left.\left.A_{1}\right\},\left\{Q_{2}, A_{2}\right\}, \ldots,\left\{Q_{n}, A_{n}\right\}\right\}\right)$. The forward propagation process iterates through all previous turns and finally estimates the loss values for $A_{n}$ and $Q_{n}$. In this process, we pass the GPT-based $(\mathrm{K}, \mathrm{V})$ representation forward to the next module, which accumulates the representations of each pre- vious turn, with the original highlighted passage as reference. For each sub-dialogue, we only update the loss from the answer and question in the last turn since the highlighted span specifies the information for the last turn. For a sub-dialog of $n$ turns, the loss is calculated as

$$
\text { Loss }=\operatorname{Loss}_{A_{n}}+\operatorname{Loss}_{Q_{n}}
$$

where

$$
\operatorname{Loss}_{A_{n}}=C E\left(A_{n}, P_{A_{n}}\right)
$$

and

$$
\operatorname{Loss}_{Q_{n}}=C E\left(Q_{n}, P_{Q_{n}}\right)
$$

$\mathrm{CE}$ refers to the cross-entropy loss from a target sentence. The parameters of the model are updated by backpropagating the aggregated loss values. By considering the encoding of the previous turns for estimating the loss and increasingly considering various sub-dialogues, the flow propagation-based training improves the conversational flow of the CQG.

\section{Experiments}

\subsection{Dataset}

We conduct experiments on the CoQA dataset (Reddy et al., 2019), which is a large-scale conversational question answering dataset composed of $8 \mathrm{k}$ conversations with $127 \mathrm{k}$ question-answer pairs collected via Amazon Mechanical Turk. Each dialogue turn also contains the supporting rationale for each answer. A number of different question types 
are present, as documented in the original paper, including Yes/No, explanation (i.e. How?, Why?), and factoid (i.e. What? When? Where? How much?). Yes/No and explanation questions, alongside the overall conversational language, make this an exceedingly challenging dataset for CQG. Since the private test set is not available, we conduct experiments on the training set and the dev set. We randomly sample $10 \%$ from the original training set to form the test set, and keep the original dev set unchanged. We conduct our experiments with a training set with 97783 examples, dev set with 7983 examples and test set with 10846 examples. We report the performance on the test set.

\subsection{Implementation Details}

We use both $\mathrm{GPT}_{\text {small }}$ and $\mathrm{GPT}_{\text {medium }}$ in all experiments. For baselines, we consider ReDR, the SOTA method in answer-unaware CQG, and CFNet, the SOTA method in answer-aware CQG. We also implemented two SOTA pre-training generation models, T5 and BART. They utilize all our preprocessing methods and training skills except the AE/QG modules. We used $\mathrm{T} 5_{\text {large }}(770 \mathrm{M})$ and $\mathrm{BART}$ large (400M), which are comparable with ChainCQG-M in terms of parameter size.

We initialize ChainCQG with the open sourced GPT-2 parameters (Radford et al., 2019). We apply AdamW optimizer (Loshchilov and Hutter, 2019), and the warmup ratio is set to 0.1 . The learning rate is tuned between $2 \mathrm{e}-5$ and $5 \mathrm{e}-5$. The dropout ratio is set to be 0.1 . We decode questions by nucleus sampling (Holtzman et al., 2020) with top-p as 0.2, top-k as 400 , and temperature as 0.7 .

\subsection{Evaluation Metric}

Our main objectives when evaluating our model are quality of the generated questions and performance on our task goal (e.g., asking conversational questions that are consistent with the target answers). To this end, we first examine a set of automated metrics. Then, to ensure robustness, we evaluate and discuss a set of human-based metrics.

\subsubsection{Automated Metrics}

To evaluate our question generation approach, we aim to show that it is 1) grammatically and semantically correct and 2) able to achieve the task objectives. To achieve the first goal, we compute automatic metrics with respect to the ground truth questions. We report multiple commonly used metrics, including BLEU (Papineni et al., 2002),
ROUGE (Lin, 2004), METEOR (Banerjee and Lavie, 2005), and perplexity (Clarkson and Robinson, 1999). BertScore (Zhang* et al., 2020) and MoverScore (Zhao et al., 2019) are recently proposed metrics that utilize a large pre-trained model to evaluate the generation quality at the semantic level. We use both of these to evaluate the semantic similarity between the generated question and the reference question. Since two questions could express similar meaning with low lexical overlap, these two semantic-level metric could also show important information about the question quality.

\subsubsection{Human Evaluation Metrics and Procedure}

In this section, we discuss the metrics used for human evaluation. Human evaluation provides additional support for the approach and the robustness of automatic evaluation. Specifically, we use answerability and fluency to measure the quality of the generated questions in relation to the context. We have used Mechanical Turk for this evaluation.

In the context of answer-aware question generation, Answer Consistency describes whether the generated questions result in the correct answers (Celikyilmaz et al., 2020). To measure this metric, we provide the passage and the answer, and ask the evaluators whether the generated question is consistent with the answer (i.e., 1 for consistent and 0 for inconsistent). Fluency measures the quality of the generated questions and accounts for criteria such as grammar, spelling, choice of words, and style (Du et al., 2017). To measure this metric, we provide the generated question and ask the human evaluator whether the language in the generated question is fluent. We consider three categories of errors: grammar/spelling mistakes, missing entity names, and mismatched pronouns. Based on these categories, we assign 2 for cases with no mistake in any of the categories, 1 for cases with maximum of one mistake in any of the mentioned categories, and 0 for cases with one or more mistakes in each one of the categories. We scale the fluency score to $(0,1)$ by maximum evaluation scores.

\subsection{Main Results}

The ChainCQG model architecture is evaluated alongside two SOTA baselines (ReDR, CFNet) on a number of automatic metrics, including BLEU (1-4), METEOR, ROUGE-L, MoverScore, and BERTScore. More information about these metrics and baselines was presented in Section 4.3. 


\begin{tabular}{ccccccccc}
\hline Model & B1 & B2 & B3 & B4 & M & RL & BS & MS \\
\hline ReDR & 27.58 & 7.81 & 2.83 & 1.35 & 12.15 & 34.05 & 87.14 & 7.62 \\
\hline CFNet & 38.24 & 22.60 & 16.11 & 12.23 & 25.75 & 43.25 & 91.25 & 25.92 \\
\hline BART-large & 49.41 & 30.57 & 19.40 & 12.34 & 35.78 & 46.88 & 92.55 & 31.89 \\
\hline T5-large & 50.83 & 32.64 & 20.81 & 13.84 & 37.08 & 48.67 & 92.86 & 33.91 \\
\hline ChainCQG-M & $\mathbf{5 3 . 1 5}$ & $\mathbf{3 5 . 3 1}$ & $\mathbf{2 3 . 3 1}$ & $\mathbf{1 5 . 7 8}$ & $\mathbf{4 0 . 1 5}$ & $\mathbf{5 0 . 9 8}$ & $\mathbf{9 3 . 1 4}$ & $\mathbf{3 6 . 4 0}$ \\
ChainCQG-S & 49.26 & 31.06 & 20.24 & 12.11 & 33.26 & 46.23 & 92.53 & 32.82 \\
\hline
\end{tabular}

Table 1: Automated Metric Evaluation Results.

These scores seek to evaluate the lexical overlap, and to some degree, the semantic similarity, between generated and ground truth questions within each dialogue turn. We also train two QG models based on pretrained Transformer seq2seq architectures (BART-large (Lewis et al., 2020), T5large (Raffel et al., 2020)) using all elements of the ChainCQG methodology except the questionanswer representation sharing mechanism used in Chain CQG model. Instead, the target answer is the direct input to the model, concatenated with the passage and dialogue history.

\subsubsection{Automated Metrics Results}

Results of all models and baselines are shown in Table 1. In the top row of this Table, $\mathrm{P}$ is the perplexity, B1-4 are BLEU 1 through BLEU 4, $\mathrm{M}$ is METEOR, RL is ROUGE-L, BS is the BERTScore, and MS is MoverScore. In the first column, ChainCQG-M and ChainCQG-S refer to two version of our approach using medium and small GPT-2. Major observations are listed below:

The top performing ChainCQG model, composed of two GPT-2 Medium modules, improves upon all baselines by a considerable margin and across all the considered metrics. In addition, it also outperforms the T5-large, which has more parameters, by a large margin. This suggests that the ChainCQG learns a better representation using the AE-QG structure, with less parameters than the T5. We improve upon the current answeraware CQG SOTA, CFNet, on each metric as well. Note that with our methods, T5-large and BARTlarge also outperforms the SOTA methods. T5large is the next best performing model, trailing Chain $C Q G$ by at least two points on all metrics except BERTScore, which shows a narrower margin of improvement.

\subsubsection{Human Evaluation Results}

It is well-known that automatic evaluation metrics do not always correlate with human judgement in

\begin{tabular}{ccc}
\hline Model & Consistency & Fluency \\
\hline CFNet & 0.710 & 0.439 \\
\hline BART & 0.792 & 0.482 \\
\hline T5 & 0.757 & 0.462 \\
\hline ChainCQG-M & $\mathbf{0 . 8 1 7}$ & $\mathbf{0 . 5 4 8}$ \\
\hline
\end{tabular}

Table 2: Human Evaluation Results.

conversational generation tasks (Celikyilmaz et al., 2020). Especially in the context of CQG, there is a many-to-one relationship between questions and their target answers and dialogue contexts, and token-based metrics are inherently unable to measure the similarity between such sequences with low degrees of lexical overlap. As a recourse, we also assess our model performance on a number of human evaluation metrics described in a previous section: Answer Consistency and Fluidity. These metrics cover an important cross-section of human judgement, which is not represented in the automatic metrics. Specifically, we seek to quantify the naturalness and consistency of Chain $C Q G$ results within each dialogue span. Table 2 shows the performance of our models and baselines on these metrics.

\subsection{Results Discussion}

Overall, both standalone QG models using BART and T5, as well as ChainCQG outperformed the SOTA baseline, CFNet, on both metrics, while the Chain $C Q G$ model achieved the best performance of all models on both metrics. The Answer Consistency roughly indicates that the question types were better aligned with the target answer and dialogue history, than the baseline, while the Fluency metric points to improvements in factors like grammar, coreference alignment, and dialogue flow. Together with the Automatic Metrics, these results support our finding that the ChainCQG model is able to learn to produce conversational dialogue that is well aligned with the CoQA dataset, both lexically 
and semantically, and more robust in general.

The improvement over single CQG seq2seq models like T5 demonstrates the success of learning joint question-answer representations and using the encoding of the latter to inform the QG module. We present a more complete analysis of error and ablation studies in the following sections. The answer-aware QG strategy is also validated here, as shown by the significant improvement of every answer-aware model over the answerunaware (ReDR) baseline. Finally, the comparison of ChainCQG to the current answer-aware QG SOTA, CFNet, demonstrates the importance of the question-answer representation and encoding scheme in our model. While CFNet incorporates tactical model components to improve the quality of CQG along specific dimensions, such as coreference and dialogue flow, our model is able to flexibly learn the progression of questions without the need for architectural components that target specific dialogue features. Another important point is that CFNet excluded all Yes/No questions from their analysis, as they proved difficult to reliably generate. Our model not only achieves SOTA performance, but is also able to natively generate every question type present in the dataset. Moreover, we notice that our model shows improved coreference alignment ability when generating questions with complex and entangled dialog history.

\subsection{Ablation Study}

Our ablation study aims to understand the effectiveness of various design choices in the Chain $C Q G$ approach outlined (in Section 3.3). In all ablation experiments, the reference model is the Chain $C Q G$ model, combining the AE and QG modules. Results of this analysis are shown in Table 3. We have applied the following ablations:

\subsubsection{Removing the dialogue history}

Here, we evaluate the effect of the flow propagation training scheme. Table 3 shows that removing the dialogue history, and consequently, any notion of dialogue flow, reduces performance across all the metrics (e.g., approximately $14 \%$ for both small and medium versions). These results match the intuition that dialogue history provides essential context to correctly handle coreferences and natural transitions.

\subsubsection{Removing the answer rationale highlight tokens}

To evaluate the effect of grounding the generated questions in the relevant passage text, we remove the answer rationale highlight tokens from the input passage. The results in Table 3 show that this ablation decreases performance in all the metrics. For example, removing the highlight reduces BLEU1 for the medium GPT from 53.15 to 47.07 (approximately $11 \%$ reduction). We conclude that the highlighted tokens ground the model in the relevant passage information, providing essential context while focusing the scope of the question.

\subsubsection{Changing the order of the questions and answers}

As discussed in Section 3.2, we have used the AQ order instead of QA in our input encoding. Here, we evaluate the effect of such ordering. As Table 3 shows, reversing the order of the question and answers results in a performance reduction of approximately $5 \%$ in the BLEU- 1 score for the medium GPT model. This shows that the AQ order is a more natural structure for dialogue flow propagation, since answers precede the generated question in each turn.

\subsubsection{Removing the AE module}

As discussed in Sections 1 and 3.3, by including the $\mathrm{AE}$ module, we aim to address the challenge of expressing the representations of questions and answers over multiple dialogue turns. Here, we remove the AE module to validate the effect of this modelling choice. The results in Table 3 show that removing the AE module reduces the performance of the model by $3 \%$ and $8 \%$ in the BLEU1 score for the medium and small GPT models, respectively. This indicates that propagating the question-answer representations across dialogue turns produces rich temporal representations that improve the fidelity of dialogue flow.

\subsection{Error Analysis}

In order to better understand the performance differentiation between our model and baselines considered here, we inspected some samples of generated questions with poor quality. While the SOTA baseline, CFNet, neglected all Yes/No questions completely, our model is overall, very successful at generating this type, alongside others such as factoid and explanation. However, Yes/No questions 


\begin{tabular}{cccccccccc}
\hline Model & P & B1 & B2 & B3 & B4 & M & RL & BS & MS \\
\hline ChainCQG-M & 7.04 & 53.15 & 35.31 & 23.31 & 15.78 & 40.15 & 50.98 & 93.14 & 36.40 \\
ChainCQG-S & 9.55 & 49.26 & 31.06 & 20.24 & 12.11 & 33.26 & 46.23 & 92.53 & 32.82 \\
\hline M w/o history & 9.13 & 45.53 & 28.35 & 18.31 & 11.27 & 30.35 & 40.45 & 92.54 & 27.59 \\
S w/o history & 11.1 & 42.54 & 25.63 & 14.91 & 8.01 & 27.73 & 39.23 & 91.03 & 24.30 \\
\hline M w/o highlight & 7.83 & 47.07 & 30.63 & 20.54 & 12.91 & 32.56 & 43.63 & 92.36 & 31.50 \\
S w/o highlight & 11.09 & 43.63 & 25.74 & 17.14 & 10.54 & 27.73 & 40.43 & 91.23 & 24.82 \\
\hline M w/o AQ order & 7.43 & 50.23 & 33.65 & 21.43 & 14.08 & 37.35 & 48.88 & 92.94 & 34.73 \\
S w/o AQ order & 9.90 & 47.65 & 30.51 & 19.04 & 10.80 & 31.58 & 42.82 & 92.21 & 29.74 \\
\hline M w/o AE module & 8.05 & 51.64 & 33.26 & 21.26 & 13.86 & 37.23 & 47.23 & 92.64 & 32.23 \\
S w/o AE module & 15.21 & 45.23 & 28.19 & 18.01 & 10.73 & 29.43 & 44.12 & 92.13 & 27.28 \\
\hline
\end{tabular}

Table 3: Ablation Study Results.

can still be problematic when the answer context includes many potential targets, each of which could be satisfied by a consistent Yes/No question. We also find that in minority cases, ChainCQG cannot handle questions requiring complex logic or reasoning to arrive at the target answer. We hypothesize that a more powerful pre-training model could alleviate this issue. Also, the Chain $C Q G$ model sometimes includes additional details related to the answer, not contained in the gold question, which results in slightly more verbose, though consistent, questions.

\section{Discussion and Ethical Issues}

The results presented here demonstrate the efficacy of modern Transformer-based architectures, and specifically Chain $C Q G$, in producing conversational questions on a challenging dataset. While answer-aware QG is our focus here, we plan to expand this in future work to include answer-unaware and open-ended QG, multi-task NLG involving $\mathrm{QA}$, and domain-specific dialogue simulation. The flexibility of the Chain $C Q G$ architecture lends itself well to each of these problems, as representations from different inputs and tasks can be shared easily between modules.

As for the practical implications of our QG work, a number of applications mentioned in previous sections could immediately take advantage of QG features, either for training QA models or generating user-facing questions. In the former setting, generation models, such as ChainCQG, risk polluting the training dataset with examples that are noisy or inconsistent with the target answers, which can cause undesirable effects at inference time. In the latter setting, generation models may suffer from bias based on the questions available for training, which may lead to misrepresentation of application domains and individual users. Additional work is required to understand the extent of these issues in real-world applications, and identify corrective measures to ensure model robustness and diversified training distributions.

\section{Conclusion}

In this paper, we introduce ChainCQG, an answeraware Conversational Question Generation model that outperforms all baselines on both automatic and human evaluation metrics on the inverted CoQA dataset (e.g., BLEU-1 improvement of $48 \%$ and $28 \%$ with GPT medium compared to ReDR and CFNet, respectively). We have designed a twostage GPT-2-based architecture that jointly learns passage and dialogue history representations via a flow propagation training method. Chain $C Q G$ produces high-quality questions in multi-turn dialogue, addressing previous SOTA issues such as question type fidelity, question-answer inconsistency and coreference misalignment. Finally, we have performed and presented extensive ablation studies for various aspects of our approach.

\section{References}

Satanjeev Banerjee and Alon Lavie. 2005. Meteor: An automatic metric for $\mathrm{mt}$ evaluation with improved correlation with human judgments. In Proceedings of the acl workshop on intrinsic and extrinsic evaluation measures for machine translation and/or summarization, pages 65-72.

Paweł Budzianowski, Tsung-Hsien Wen, Bo-Hsiang Tseng, Iñigo Casanueva, Stefan Ultes, Osman Ramadan, and Milica Gašić. 2018. MultiWOZ - a large-scale multi-domain Wizard-of-Oz dataset for task-oriented dialogue modelling. In Proceedings of 
the 2018 Conference on Empirical Methods in Natural Language Processing, pages 5016-5026, Brussels, Belgium. Association for Computational Linguistics.

Asli Celikyilmaz, Elizabeth Clark, and Jianfeng Gao. 2020. Evaluation of text generation: A survey.

Yu Chen, Lingfei Wu, and Mohammed J. Zaki. 2020. Graphflow: Exploiting conversation flow with graph neural networks for conversational machine comprehension. In Proceedings of the Twenty-Ninth International Joint Conference on Artificial Intelligence, IJCAI-20, pages 1230-1236. International Joint Conferences on Artificial Intelligence Organization. Main track.

Eunsol Choi, He He, Mohit Iyyer, Mark Yatskar, Wentau Yih, Yejin Choi, Percy Liang, and Luke Zettlemoyer. 2018. QuAC: Question answering in context. In Proceedings of the 2018 Conference on Empirical Methods in Natural Language Processing, pages 2174-2184, Brussels, Belgium. Association for Computational Linguistics.

Philip Clarkson and Tony Robinson. 1999. Towards improved language model evaluation measures. In Sixth European Conference on Speech Communication and Technology.

Xinya Du and Claire Cardie. 2017. Identifying where to focus in reading comprehension for neural question generation. In Proceedings of the 2017 Conference on Empirical Methods in Natural Language Processing, pages 2067-2073.

Xinya Du and Claire Cardie. 2018. Harvesting paragraph-level question-answer pairs from Wikipedia. In Proceedings of the 56th Annual Meeting of the Association for Computational Linguistics (Volume 1: Long Papers), pages 1907-1917, Melbourne, Australia. Association for Computational Linguistics.

Xinya Du, Junru Shao, and Claire Cardie. 2017. Learning to ask: Neural question generation for reading comprehension. In Association for Computational Linguistics $(A C L)$.

Layla El Asri, Hannes Schulz, Shikhar Sharma, Jeremie Zumer, Justin Harris, Emery Fine, Rahul Mehrotra, and Kaheer Suleman. 2017. Frames: a corpus for adding memory to goal-oriented dialogue systems. In Proceedings of the 18th Annual SIGdial Meeting on Discourse and Dialogue, pages 207219, Saarbrücken, Germany. Association for Computational Linguistics.

Yifan Gao, Piji Li, Irwin King, and Michael R. Lyu. 2019. Interconnected question generation with coreference alignment and conversation flow modeling. In Proceedings of the 57th Annual Meeting of the Association for Computational Linguistics, pages 4853-4862, Florence, Italy. Association for Computational Linguistics.
Jing Gu, Qingyang Wu, Chongruo Wu, Weiyan Shi, and Zhou Yu. 2020. A tailored pre-training model for task-oriented dialog generation.

Ari Holtzman, Jan Buys, Li Du, Maxwell Forbes, and Yejin Choi. 2020. The curious case of neural text degeneration. In International Conference on Learning Representations.

Hsin-Yuan Huang, Eunsol Choi, and Wen tau Yih. 2019. FlowQA: Grasping flow in history for conversational machine comprehension. In International Conference on Learning Representations.

Ying Ju, Fubang Zhao, Shijie Chen, Bowen Zheng, Xuefeng Yang, and Yunfeng Liu. 2019. Technical report on conversational question answering. arXiv preprint arXiv:1909.10772.

Yanghoon Kim, Hwanhee Lee, Joongbo Shin, and Kyomin Jung. 2019. Improving neural question generation using answer separation. In Proceedings of the AAAI Conference on Artificial Intelligence, volume 33, pages 6602-6609.

Mike Lewis, Yinhan Liu, Naman Goyal, Marjan Ghazvininejad, Abdelrahman Mohamed, Omer Levy, Veselin Stoyanov, and Luke Zettlemoyer. 2020. BART: Denoising sequence-to-sequence pretraining for natural language generation, translation, and comprehension. In Proceedings of the 58th Annual Meeting of the Association for Computational Linguistics, pages 7871-7880, Online. Association for Computational Linguistics.

Jingjing Li, Yifan Gao, Lidong Bing, Irwin King, and Michael R. Lyu. 2019. Improving question generation with to the point context. In Proceedings of the 2019 Conference on Empirical Methods in Natural Language Processing and the 9th International Joint Conference on Natural Language Processing (EMNLP-IJCNLP), pages 3216-3226, Hong Kong, China. Association for Computational Linguistics.

Chin-Yew Lin. 2004. Rouge: A package for automatic evaluation of summaries. In Text summarization branches out, pages 74-81.

Ilya Loshchilov and Frank Hutter. 2019. Decoupled weight decay regularization. In International Conference on Learning Representations.

Mao Nakanishi, Tetsunori Kobayashi, and Yoshihiko Hayashi. 2019. Towards answer-unaware conversational question generation. In Proceedings of the 2nd Workshop on Machine Reading for Question Answering, pages 63-71.

Yasuhito Ohsugi, Itsumi Saito, Kyosuke Nishida, Hisako Asano, and Junji Tomita. 2019. A simple but effective method to incorporate multi-turn context with BERT for conversational machine comprehension. In Proceedings of the First Workshop on NLP for Conversational AI, pages 11-17, Florence, Italy. Association for Computational Linguistics. 
Boyuan Pan, Hao Li, Ziyu Yao, Deng Cai, and Huan Sun. 2019. Reinforced dynamic reasoning for conversational question generation. In Proceedings of the 57th Conference of the Association for Computational Linguistics, pages 2114-2124.

Kishore Papineni, Salim Roukos, Todd Ward, and WeiJing Zhu. 2002. Bleu: a method for automatic evaluation of machine translation. In Proceedings of the 40th annual meeting of the Association for Computational Linguistics, pages 311-318.

Peng Qi, Yuhao Zhang, and Christopher D. Manning. 2020. Stay hungry, stay focused: Generating informative and specific questions in information-seeking conversations.

Alec Radford, Jeff Wu, Rewon Child, David Luan, Dario Amodei, and Ilya Sutskever. 2019. Language models are unsupervised multitask learners.

Colin Raffel, Noam Shazeer, Adam Roberts, Katherine Lee, Sharan Narang, Michael Matena, Yanqi Zhou, Wei Li, and Peter J. Liu. 2020. Exploring the limits of transfer learning with a unified text-totext transformer. Journal of Machine Learning Research, 21(140):1-67.

Pranav Rajpurkar, Jian Zhang, Konstantin Lopyrev, and Percy Liang. 2016. SQuAD: 100,000+ questions for machine comprehension of text. In Proceedings of the 2016 Conference on Empirical Methods in Natural Language Processing, pages 2383-2392, Austin, Texas. Association for Computational Linguistics.

Siva Reddy, Danqi Chen, and Christopher D Manning. 2019. Coqa: A conversational question answering challenge. Transactions of the Association for Computational Linguistics, 7:249-266.

Thomas Scialom, Benjamin Piwowarski, and Jacopo Staiano. 2019. Self-attention architectures for answer-agnostic neural question generation. In Proceedings of the 57th Annual Meeting of the Association for Computational Linguistics, pages 6027 6032 .

Sandeep Subramanian, Tong Wang, Xingdi Yuan, Saizheng Zhang, Adam Trischler, and Yoshua Bengio. 2018. Neural models for key phrase extraction and question generation. In Proceedings of the Workshop on Machine Reading for Question Answering, pages 78-88, Melbourne, Australia. Association for Computational Linguistics.

Yansen Wang, Chenyi Liu, Minlie Huang, and Liqiang Nie. 2018. Learning to ask questions in opendomain conversational systems with typed decoders. In Proceedings of the 56th Annual Meeting of the Association for Computational Linguistics (Volume 1: Long Papers), pages 2193-2203, Melbourne, Australia. Association for Computational Linguistics.

Rainer Winkler, Sebastian Hobert, Antti Salovaara, Matthias Söllner, and Jan Marco Leimeister. 2020.
Sara, the lecturer: Improving learning in online education with a scaffolding-based conversational agent. In Proceedings of the $2020 \mathrm{CHI}$ Conference on $\mathrm{Hu}$ man Factors in Computing Systems, pages 1-14.

Qingyang Wu, Yichi Zhang, Yu Li, and Zhou Yu. 2019. Alternating roles dialog model with large-scale pretrained language models. arXiv, pages arXiv-1910.

Xiuyu Wu, Nan Jiang, and Yunfang Wu. 2020. A question type driven and copy loss enhanced frameworkfor answer-agnostic neural question generation. In Proceedings of the Fourth Workshop on Neural Generation and Translation, pages 69-78, Online. Association for Computational Linguistics.

Yi-Ting Yeh and Yun-Nung Chen. 2019. FlowDelta: Modeling flow information gain in reasoning for conversational machine comprehension. In Proceedings of the 2nd Workshop on Machine Reading for Question Answering, pages 86-90, Hong Kong, China. Association for Computational Linguistics.

Tianyi Zhang*, Varsha Kishore*, Felix Wu*, Kilian Q. Weinberger, and Yoav Artzi. 2020. Bertscore: Evaluating text generation with bert. In International Conference on Learning Representations.

Wei Zhao, Maxime Peyrard, Fei Liu, Yang Gao, Christian M. Meyer, and Steffen Eger. 2019. MoverScore: Text generation evaluating with contextualized embeddings and earth mover distance. In Proceedings of the 2019 Conference on Empirical Methods in Natural Language Processing and the 9th International Joint Conference on Natural Language Processing (EMNLP-IJCNLP), pages 563-578, Hong Kong, China. Association for Computational Linguistics.

Yao Zhao, Xiaochuan Ni, Yuanyuan Ding, and Qifa Ke. 2018. Paragraph-level neural question generation with maxout pointer and gated self-attention networks. In Proceedings of the 2018 Conference on Empirical Methods in Natural Language Processing, pages 3901-3910.

Qingyu Zhou, Nan Yang, Furu Wei, Chuanqi Tan, Hangbo Bao, and Ming Zhou. 2017. Neural question generation from text: A preliminary study. In National CCF Conference on Natural Language Processing and Chinese Computing, pages 662-671. Springer.

Chenguang Zhu, Michael Zeng, and Xuedong Huang. 2018. Sdnet: Contextualized attention-based deep network for conversational question answering. arXiv preprint arXiv:1812.03593. 